\title{
La auriculopuntura disminuye los niveles de ansiedad en adultos chilenos con malnutrición por exceso
}

\author{
Auriculopuncture decreases anxiety levels in Chilean adults with malnutrition by excess
}

Eduard Maury-Sintjago ${ }^{*}$ orcid.org/0000-0002-9482-8268

Javiera Robledo-Larenas ${ }^{2}$ orcid.org/0000-0003-1908-3996

Jennifer Pinto-Gallardo² orcid.org/0000-0001-8775-3723

Alejandra Rodríguez-Fernández1 orcid.org/0000-0002-3982-3165

1 Departamento de Nutrición y Salud Pública, Facultad Ciencias de la Salud y de los Alimentos, Universidad del Bío-Bío. Chillán, Chile

2 Carrera de Nutrición y Dietética, Facultad de Salud y Ciencias de la Actividad Física, Universidad SEK. Santiago, Chile

\section{Resumen}

Introducción: A pesar del incremento en el uso de la medicina tradicional china para el tratamiento de la obesidad son pocos los estudios que evalúan su efecto. Objetivo: Determinar el efecto de la auriculopuntura sobre la ansiedad, ingesta nutricional y medidas antropométricas en chilenos con malnutrición por exceso. Materiales y métodos: estudio cuasi-experimental, con muestra probabilística conformada por 21 sujetos, divididos en dos grupos, intervenido GI $(\mathrm{n}=11)$ y control GC $(\mathrm{n}=10)$. La intervención consistió en realizar auriculopuntura durante siete semanas al GI y administrar un placebo al GC. En ambos grupos se determinó antropometría, ingesta nutricional y niveles de ansiedad. Todos los participantes firmaron consentimiento informado. Para comparar medias entre grupos e intra grupos, se utilizó la prueba t-Student para muestras pareadas. La significancia estadística se estableció a un $(\alpha<0,05)$. Resultados: Se encontró en el GI una disminución de la ansiedad $(p=0,036)$ y de la ingesta de sodio $(p=0,035)$. No se encontraron diferencias estadísticamente significativas en las variables de ingesta calóricanutricional y antropométrica en ningún grupo. Conclusiones: Es necesario incrementar el tiempo de intervención para evaluar el efecto en el largo plazo y de esta forma, establecer asociaciones causales.

Palabras clave: Ansiedad; antropometría; auriculoterapia. (Fuente: DeCS, Bireme).

\begin{abstract}
Introduction: Despite the increase in the use of traditional Chinese medicine for the treatment of obesity, there are few studies which evaluate its effect. Objective: To determine the effect of auriculopuncture on anxiety, nutritional intake and anthropometric measures in Chilean adults with malnutrition by excess. Materials and methods: A quasi-experimental study was conducted with a probabilistic sample conformed by 21 subjects, divided into two groups: intervened GI $(\mathrm{n}=11)$ and control GC $(\mathrm{n}=10)$. The intervention consisted in performing auriculopuncture for seven weeks to GI and administering a placebo to GC. In both groups anthropometry, nutritional intake and anxiety levels were determined. All participants signed informed consent. To compare averages between groups and intra groups, the T-student test was used for paired samples. Statistical significance was set to one $(\alpha<0.05)$. Results: A decrease in anxiety $(p=0.036)$ and sodium intake $(p=0.035)$ was found in the GI. No statistically significant differences were found in the variables of caloric-nutritional and anthropometric intake in any group. Conclusions:
\end{abstract}

*Autor de correspondencia

Eduard Maury Sintjago

e-mail: emaury@ubiobio.cl 
It is necessary to increase the intervention time to evaluate the long-term effect and thus establish causal associations.

Key words: Anxiety; anthropometry; auriculotherapy. (Source: DeCS, Bireme).

\section{Introducción}

No se puede refutar el incremento exponencial que han tenido los establecimientos que ofertan servicios de medicina complementaria alternativa para el tratamiento de diversas patologías físicas o mentales, e incluso para la promoción de salud y prevención de enfermedades $^{(1)}$.

La medicina complementaria alternativa es definida como el conjunto de prácticas y productos médicos que se ubican en una esfera alternativa de la medicina convencional. Dentro de estas podemos encontrar la medicina ayurveda hindú, la unani (perso-árabe) y la medicina tradicional china (MTCH); que es la medicina complementaria alternativa más utilizada en la esfera mundial(2). El informe de estrategia sobre medicina tradicional de la Organización Mundial de la Salud (OMS), da cuenta de que en algunas regiones del mundo es utilizada por $40-80 \%$ de la población para satisfacer sus demandas sanitarias ${ }^{(3)}$.

En Chile, el Ministerio de Salud, desde el año 2005 ha reconocido e implementado el ejercicio de las prácticas médicas alternativas, como auxiliares a las prácticas médicas propias, entre ellas; la homeopatía, naturopatía y la acupuntura. Esta última es un componente fundamental de la MTCH que consiste en la inserción y manipulación de agujas en el cuerpo ejerciendo presión en puntos específicos con el objetivo de restaurar la salud y el bienestar en el paciente(4).

De la acupuntura deriva una importante rama, la auriculopuntura, que considera la existencia de hologramas o planos de la totalidad del cuerpo y sus zonas más distales en el pabellón auricular(5). La técnica consiste en realizar estímulos (con semillas, laser, electricidad, ajugas y otros) en puntos específicos del holograma auricular para tratar patologías. Dentro de las enfermedades más tratadas por la auriculoterapia se encuentran cefaleas, cáncer, lumbalgias, ansiedad, obesidad, entre otros(6).

La obesidad es un importante problema de la salud pública en Chile y el mundo, significa tanto un costo económico como social para las personas y el Estado. Su prevalencia ha ido en aumento según la última Encuesta Nacional de Salud del año 2016-2017, donde el exceso de peso del año 2009-2010 aumentó de un 67\% a un $74 \%$ en la población mayor de 15 años(7). La obesidad es una enfermedad multifactorial, por tanto sus causas etiológicas son muy diversas que van desde aspectos genéticos, falta de actividad física, concomitancia de alguna patología, desbalance energético y aspectos psicológicos(8). Existe una asociación entre la ansiedad, la ingesta calórica-energética y la obesidad( $(9)$.

Da Silva presentó un estudio de caso en el que se logró disminuir los síntomas de ansiedad con acupuntura tradicional china en una intervención que duró 10 sesiones(10). Por otro lado, Haddad y Marcon muestran en su estudio con obesos brasileños que hubo cambios en la ansiedad, plenitud e ingesta de alimentos tras ocho sesiones de acupuntura(11). Sin embargo, Set et al., en su estudio con mujeres turcas con malnutrición por exceso, reportó que tras una intervención de 6 sesiones de auriculoterapia no se encontró diferencias significativas en la ingesta dietética y los niveles de ansiedad(12).

El actual incremento de la medicina complementaria alternativa principalmente la MTCH, aunado por el incremento de la malnutrición por exceso a nivel poblacional y de los posibles efectos que tienen la acupuntura y la auriculopuntura en el tratamiento de la obesidad convoca al aumento de la evidencia científica. Por ello, el objetivo del presente estudio es determinar el efecto de la auriculopuntura sobre niveles de ansiedad, ingesta calórica-nutricional y medidas antropométricas en adultos chilenos. 


\section{Materiales y métodos}

Este es un estudio de tipo cuasi experimental. La muestra se calculó en base a la comparación de medias, donde se consideró un nivel de confianza del $95 \%$ y una potencia del $90 \%$. La varianza y diferencia de medias máxima usada se basó en el estudio de Viner et al.(13). De esta forma, quedó constituida por 21 adultos de ambos sexo, entre 18 y 65 años, con malnutrición por exceso (IMC $\geq 25$ ). El reclutamiento de los sujetos de estudio se realizó mediante convocatoria con afiches donde se invitaba a participar del estudio. Con todos los participantes reunidos, se procedió a presentar el estudio en términos de sus objetivos, procedimientos y alcances.

Se excluyeron los participantes que reportaron alguna enfermedad endocrina, cardiaca, alérgica, inmunológica, antecedentes de diabetes, mujeres embarazadas, o en periodo de lactancia o aquellas que habían dado a luz en los últimos seis meses, ni aquellos quienes a la fecha del estudio consumían medicamentos.

La conformación de los grupos intervenido (GI) y control (GC), se realizó distribuyendo de manera aleatoria 11 sujetos para el GI y 10 para el GC. Como control de enmascaramiento los participantes desconocían el grupo al cual fueron asignados. El GI fue aquel al que se le realizó intervención con auriculopuntura, y al GC se le aplicó un tratamiento placebo.

La intervención consistió en aplicar sesiones de auriculopuntura durante 7 semanas al GI estimulando los puntos que en el holograma auricular representan los siguientes sistemas $\mathrm{y} / \mathrm{u}$ órganos: shenmen, sistema simpático, boca, duodeno, estómago, sed, hambre, glándula de secreción y punto cero, para el tratamiento de la ansiedad, ingesta de alimentos y pérdida de peso(14); en la primera sesión y como única vez se estimuló el punto cero. Se utilizaron agujas intradérmicas ( $3 \mathrm{~mm}$ de longitud por $0,12 \mathrm{~mm}$ de diámetro) a una profundidad de 1-2 mm, previamente la zona fue desinfectada con etanol. Las agujas eran reemplazadas semanalmente.
El tratamiento placebo para el CG consistió en suministrar agua mineral sin gas con 16 gotas de esencia de vainilla, donde los participantes debían colocar 3 gotas debajo de la lengua con una frecuencia de 3 veces al día, durante el periodo de estudio.

Al inicio de la intervención se recolectó información demográfica (edad, sexo) y aplicó el test de Hamilton para evaluar los niveles de ansiedad, encuesta recordatorio de 24 horas y evaluación antropométrica(15). En este periodo, se entregó a ambos grupos una pauta de alimentación individualizada y educación nutricional, según el estado nutricional del paciente. En el periodo final (séptima semana), se realizó un control de peso, evaluación de ingesta y el test de Hamilton.

\section{Ingesta dietética}

La información de consumo de alimentos en los participantes se obtuvo mediante el método de recordatorio de 24 horas. Se registraron todos los alimentos y bebidas consumidos durante todas las semanas del estudio. Se utilizaron modelos de alimentos y medidas prácticas para mejorar la estimación del tamaño de las raciones. La determinación del consumo de nutrientes por día se realizó a partir de los datos de los alimentos consumidos, los cuales fueron llevados a gramos de alimentos y se calculó la composición calórica y de nutrientes, a partir de la tabla de composición de alimentos chilenos.

\section{Niveles de ansiedad}

Para determinar los niveles de ansiedad en los sujetos participantes se aplicó la escala de ansiedad de Hamilton(15). El entrevistador puntúa de 0 a 4 puntos cada ítem. Una mayor puntuación indica una mayor intensidad de la ansiedad. Esta escala es sensible a las variaciones a través del tiempo o tras recibir tratamiento.

Se han definido los puntos de corte para establecer una medida global de ansiedad. Los puntos de corte recomendados son:

0-5: no ansiedad

6-14: ansiedad leve

$\geq 15$ : ansiedad moderada/grave 
El test de Hamilton se aplicó al inicio y final de la intervención. Finalmente, para corroborar la consistencia de la Escala de Ansiedad de Hamilton en nuestro estudio, se aplicó una prueba piloto obteniendo un alfa de Cronbach de 0,985 .

\section{Evaluación antropométrica}

Se realizaron las evaluaciones antropométricas siguiendo los procedimientos normalizados. Se obtuvo valores del peso, talla e índice de masa corporal (IMC). La medición de peso se realizó en las condiciones más estandarizadas posibles; los sujetos debían estar descalzos, con un mínimo de ropa (ropa interior), la evaluación se realizó con una balanza electrónica (Tanita TBF 300A ®) con un rango de medición hasta $200 \mathrm{~kg}$ y de 0.1 kg de precisión.

La medida de la estatura se realizó utilizando un estadiómetro portátil (Marca SECA, modelo 217); para ello los sujetos se ubicaron descalzos, en bipedestación, con la espalda lo más recta posible, brazos extendidos y paralelos al cuerpo, talones juntos y cabeza colocada siguiendo el plano horizontal de Frankfort. Para el cálculo del IMC se debió realizar una ecuación matemática: peso (kg)/estatura (metros) al cuadrado. Como parámetros de referencia se utilizaron los valores propuestos por la Organización Mundial de Salud (OMS) ${ }^{(16) .}$

\section{Análisis estadístico}

Se evaluó la distribución normal de los datos utilizando la prueba de Shapiro-Wilk. Los datos se expresaron con estadística descriptiva (promedio, desviación estándar y porcentaje). Para comparar medias entre e intra grupos según tiempos de intervención se utilizó la prueba t-Student para muestras pareadas. Se estableció un nivel de significancia estadística de $\mathrm{p}<0,05$. Para el análisis estadístico se utilizó el programa estadístico SPSS Versión 21.

\section{Consideraciones bioéticas}

Los participantes lo hicieron de manera voluntaria $y$ firmaron un consentimiento informado. Se resguardaron los principios bioéticos y el anonimato de los participantes. Los procedimientos empleados fueron ejecutados de acuerdo a las normas éticas de la declaración de Helsinki y el Consejo de la Organización Internacional para las Ciencias Médicas (CIOMS) $^{(17) .}$

\section{Resultados}

El $90 \%$ de la muestra fue de sexo femenino. El $65 \%$ tenía una edad entre 21-25 años. En la Tabla 1, se observa la comparación de las variables entre los grupos de estudio, según tiempo de intervención. Al inicio de la intervención (pre-intervención) no se encontró diferencias significativas en ninguna variable medida. Al comparar los datos entre grupos en el periodo final (post-intervención), no se encontró diferencias significativas en las variables de estudio, salvo en la puntuación del test de Hamilton, siendo esta menor en GI $(7,9 \pm 3,8)$ en relación a GC $(12,8 \pm 5,6)(\mathrm{p}=0,028)$.

En la Tabla 2, se muestra la variación de las ingesta dietética, niveles de ansiedad y medidas antropométricas según los tiempos de intervención del GI. Se puede observar diferencias estadísticas significativas en el test de Hamilton $(p=0,036)$ y en el consumo de sodio $(\mathrm{p}=0,035)$, tras el tratamiento con auriculopuntura.

En relación al GC no se observan cambios estadísticamente significativos en relación a las variables estudiadas cuando se comparan según tiempo de intervención (Pre y Post-intervención) (Tabla 3). 
Tabla 1. Ingesta dietética, niveles de ansiedad y medidas antropométricas, según grupo y tiempo de intervención

\begin{tabular}{|c|c|c|c|c|c|c|}
\hline \multirow{2}{*}{ Variable } & \multicolumn{2}{|c|}{ Pre-intervención } & \multirow[b]{2}{*}{$\mathbf{p}^{*}$} & \multicolumn{2}{|c|}{ Post-intervención } & \multirow{2}{*}{ p* } \\
\hline & GI & GC & & GI & GC & \\
\hline \multicolumn{7}{|l|}{ Test de Hamilton } \\
\hline Puntuación test & $10,7 \pm 3,3$ & $14,0 \pm 3,4$ & 0,123 & $7,9 \pm 3,8$ & $12,8 \pm 5,6$ & 0,028 \\
\hline \multicolumn{7}{|c|}{ Datos antropométricos } \\
\hline Peso (Kg) & $76,5 \pm 13,1$ & $77,3 \pm 23,4$ & 0,924 & $74,9 \pm 13,2$ & $76,0 \pm 24,0$ & 0,901 \\
\hline Talla (m) & $1,6 \pm 0,1$ & $1,6 \pm 0,1$ & 1,000 & $1,6 \pm 0,1$ & $1,6 \pm 0,1$ & 1,000 \\
\hline $\mathrm{IMC}(\mathrm{Kg} / \mathrm{m} 2)$ & $29,1 \pm 4,0$ & $28,4 \pm 8,4$ & 0,804 & $28,5 \pm 4,0$ & $28,2 \pm 8,5$ & 0,907 \\
\hline \multicolumn{7}{|c|}{ Ingesta calórica y nutricional } \\
\hline Calorías (Kcal) & $1521,9 \pm 428,0$ & $1665,2 \pm 534,2$ & 0,504 & $1287,8 \pm 390,9$ & $1279,4 \pm 340,0$ & 0,959 \\
\hline Proteínas (g) & $56,3 \pm 28,3$ & $66,1 \pm 14,4$ & 0,339 & $62,0 \pm 24,1$ & $70,9 \pm 13,8$ & 0,319 \\
\hline Carbohidratos (g) & $193,8 \pm 66,2$ & $229,0 \pm 82,5$ & 0,292 & $169,0 \pm 53,0$ & $171,6 \pm 65,4$ & 0,921 \\
\hline Lípidos (g) & $47,5 \pm 32,5$ & $50,4 \pm 20,1$ & 0,813 & $40,7 \pm 19,7$ & $34,8 \pm 12,4$ & 0,423 \\
\hline Calcio (mg) & $387,4 \pm 257,6$ & $498,2 \pm 249,9$ & 0,331 & $391,6 \pm 136,4$ & $405,8 \pm 279,9$ & 0,882 \\
\hline Hierro (g) & $7,8 \pm 3,3$ & $10,3 \pm 3,6$ & 0,120 & $8,2 \pm 2,5$ & $10,0 \pm 3,4$ & 0,174 \\
\hline Zinc (g) & $4,7 \pm 2,0$ & $5,7 \pm 2,2$ & 0,264 & $4,9 \pm 2,2$ & $5,7 \pm 2,9$ & 0,481 \\
\hline Ac. Fólico $(\mu \mathrm{g})$ & $222,6 \pm 148,3$ & $302,2 \pm 207,3$ & 0,321 & $259,4 \pm 116,0$ & $303,7 \pm 163,5$ & 0,479 \\
\hline Fibra (g) & $12,9 \pm 8,1$ & $16,8 \pm 7,9$ & 0,271 & $12,2 \pm 4,2$ & $17,4 \pm 10,3$ & 0,138 \\
\hline Sodio (mg) & $1687,4 \pm 696,9$ & $1708,9 \pm 752,2$ & 0,947 & $1055,8 \pm 613,6$ & $1592,0 \pm 888,7$ & 0,121 \\
\hline
\end{tabular}

Datos expresados en promedio \pm desviación estándar.

*Prueba t-Student. Nivel de significación p<0,05

Tabla 2. Variación de ingesta dietética, niveles de ansiedad y medidas antropométricas en el grupo intervenido, según tiempo de intervención

\begin{tabular}{|c|c|c|c|}
\hline Variable & Pre-intervención & Post-intervención & $\mathbf{p}^{*}$ \\
\hline \multicolumn{4}{|l|}{ Test de Hamilton } \\
\hline Puntuación test & $10,7 \pm 3,3$ & $7,9 \pm 3,8$ & 0,036 \\
\hline \multicolumn{4}{|c|}{ Datos antropométricos } \\
\hline Peso (kg) & $76,5 \pm 13,1$ & $74,9 \pm 13,2$ & 0,784 \\
\hline IMC $\left(\mathrm{kg} / \mathrm{m}^{2}\right)$ & $29,1 \pm 4,0$ & $28,5 \pm 4,0$ & 0,723 \\
\hline \multicolumn{4}{|c|}{ Ingesta calórica y nutricional } \\
\hline Calorías (Kcal) & $1521,9 \pm 428,0$ & $1287,8 \pm 390,9$ & 0,195 \\
\hline Proteínas (g) & $53,3 \pm 28,3$ & $62,0 \pm 24,1$ & 0,620 \\
\hline Carbohidratos (g) & $193,8 \pm 66,2$ & $169,0 \pm 53,0$ & 0,343 \\
\hline Lípidos (g) & $47,5 \pm 32,5$ & $40,7 \pm 19,7$ & 0,558 \\
\hline Calcio (mg) & $387,4 \pm 257,6$ & $391,6 \pm 136,4$ & 0,962 \\
\hline Hierro (g) & $7,8 \pm 3,3$ & $8,2 \pm 2,5$ & 0,756 \\
\hline Zinc (g) & $4,7 \pm 2,0$ & $4,9, \pm 2,2$ & 0,810 \\
\hline Ac. Fólico $(\mu \mathrm{g})$ & $222,6 \pm 148,3$ & $259,4 \pm 116,0$ & 0,525 \\
\hline Fibra (g) & $12,9 \pm 8,1$ & $12,2 \pm 4,2$ & 0,808 \\
\hline Sodio (mg) & $1687,4 \pm 696,6$ & $1055,8 \pm 613,6$ & 0,035 \\
\hline
\end{tabular}


Tabla 3. Variación de ingesta dietética, niveles de ansiedad y medidas antropométricas en el grupo control, según tiempo de intervención

\begin{tabular}{|c|c|c|c|}
\hline Variable & Pre-intervención & Post-intervención & $\mathbf{p}^{*}$ \\
\hline \multicolumn{4}{|l|}{ Test de Hamilton } \\
\hline Puntuación test & $14,0 \pm 3,4$ & $12,8 \pm 5,6$ & 0,566 \\
\hline \multicolumn{4}{|c|}{ Datos antropométricos } \\
\hline Peso (kg) & $77,3 \pm 23,4$ & $76,0 \pm 24,0$ & 0,904 \\
\hline IMC $\left(\mathrm{kg} / \mathrm{m}^{2}\right)$ & $28,4 \pm 8,4$ & $28,2 \pm 8,5$ & 0,950 \\
\hline \multicolumn{4}{|c|}{ Ingesta calórica y nutricional } \\
\hline Calorías (Kcal) & $1665,2 \pm 534,2$ & $1279,4 \pm 340,0$ & 0,070 \\
\hline Proteínas (g) & $66,1 \pm 14,4$ & $70,9 \pm 13,8$ & 0,459 \\
\hline Carbohidratos (g) & $229,0 \pm 82,5$ & $171,6 \pm 65,4$ & 0,102 \\
\hline Lípidos (g) & $50,4 \pm 20,1$ & $34,8 \pm 12,4$ & 0,051 \\
\hline Calcio (mg) & $498,2 \pm 249,9$ & $405,8 \pm 279,9$ & 0,446 \\
\hline Hierro (g) & $10,3 \pm 3,6$ & $10,0 \pm 3,4$ & 0,879 \\
\hline Zinc (g) & $5,7 \pm 2,2$ & $5,7 \pm 2,9$ & 0,981 \\
\hline Ac. Fólico $(\mu \mathrm{g})$ & $302,2 \pm 207,3$ & $303,7 \pm 163,5$ & 0,986 \\
\hline Fibra (g) & $16,8 \pm 7,9$ & $17,4 \pm 10,3$ & 0,888 \\
\hline Sodio (mg) & $1708,9 \pm 752,2$ & $1592,0 \pm 888,7$ & 0,755 \\
\hline
\end{tabular}

Datos expresados en promedio \pm desviación estándar.

*Prueba t-Student. Nivel de significación $\mathrm{p}<0.05$

\section{Discusión}

La medicina complementaria y alternativa se usa cada vez más para tratar enfermedades, su aplicación ha aumentado en varios países y la tendencia es que se mantenga ${ }^{(7,18-20)}$. Asimismo, se ha iniciado un proceso de formalización de estas prácticas a través de la legislación en torno a los profesionales que la ejercen en diferentes países, entre ellos, Chile.

En este estudio se encontró que la auriculoterapia provocó una disminución estadísticamente significativa de los niveles de ansiedad $(p=0,036)$, mientras que en el grupo control no hubo cambios significativos ( $\mathrm{p}=0,566)$. Miyuki et al (2012), muestra resultados similares en su estudio con estudiantes de enfermería brasileñas tras 12 sesiones de tratamiento $(\mathrm{p}=0,03)^{(21)}$. Por otro lado, Klausenitz et al. (2016), reportan una disminución estadísticamente significativa de la ansiedad $(p=0,003)$ tras el tratamiento de auriculopuntura, pero también hubo cambios con el placebo, sin embargo el efecto de la auriculopuntura fue mayor(22).
El control de la ansiedad resulta particularmente importante en el tratamiento de sujetos con sobrepeso y obesidad. Gariepy et al. (2010), muestran en su revisión sistemática que a pesar de que existen hallazgos mixtos, en general se observa una asociación positiva entre la obesidad y la ansiedad, en 16 estudios mostró que el odds-ratio agrupado fue de 1,4 (IC=1,2$1,6)^{(23)}$. Se ha postulado que los desórdenes de ansiedad pueden ser considerados tanto una causa como una consecuencia de la obesidad(24,25).

A pesar de que se ha evidenciado que la acupuntura incrementa la liberación de dopamina y serotonina, que participan en la regulación del apetito(26), en este estudio ninguno de los grupos tuvo un cambio estadísticamente significativo en el consumo de calorías y nutrientes. Por su parte, Lacey et al., en su revisión sistemática en relación a la acupuntura para el tratamiento de la obesidad, indican que la mayoría de los ensayos clínicos que reportan cambios de ingesta han sido de corta duración, 
ensayos de naturaleza descriptiva y con protocolos no estandarizados.(27).

En relación al consumo de micronutrientes, el grupo intervenido reportó una disminución de ingesta de sodio $(\mathrm{p}=0,035)$. En un estudio hecho por Silva et al. (2013), muestran una asociación estadísticamente significativa entre la ansiedad en mujeres y el consumo de sodio(28). En esta investigación hubo una disminución de ansiedad y de consumo de sodio en el grupo intervenido además el $90 \%$ del total de la muestra eran mujeres por lo que pudiese haber una relación similar a la encontrada por Silva et al.

En ninguno de los grupos se encontró cambios en relación a las variables antropométricas aunque en ambos el promedio de kilogramos disminuyó marginalmente, similar a los resultados presentados por Hsu et al.(29), Lien et al.(30), y Ruan et al.(31). Sin embargo, difieren con otros estudios donde se ha presentado una reducción significativa de peso y/o masa grasa en sujetos tratados con auriculopuntura (32-34). En relación al control de peso o masa grasa, autores sugieren que el posible mecanismo de acción por la cual la auriculopuntura pudiese actuar es a través de una reducción de la insulina y leptina e incremento de la grelina, colecistoquinina y la expresión del neuropéptido $\mathrm{Y}^{(35-37) .}$

Es importante considerar que la diferencia entre los distintos estudios puede deberse a varios aspectos: el tiempo de tratamiento, los puntos estimulados, el estado nutricional de los sujetos y los métodos de determinación de ingesta. Asimismo, en esta investigación no se evaluó composición corporal, por lo que no se pudo evaluar el efecto que tiene la auriculopuntura en la reducción del tejido adiposo. En conclusión, bajo las condiciones del presente estudio se encontró un efecto positivo del tratamiento con auriculoterapia en los niveles de ansiedad e ingesta de sodio, pero no en las variables antropométricas y la ingesta de energía y nutrientes; aparentemente en este grupo de estudio los niveles de ansiedad no estaban relacionados con el control de ingesta dietética y por tanto con el peso corporal.

\section{Conflictos de intereses}

Los autores declaran no tener conflictos de intereses.

\section{Referencias}

1. Clarke TC, Black LI, Stussman BJ, Barnes PM, Nahin RL. Trends in the Use of Complementary Health Approaches Among Adults: United States, 2002-2012. National health statistics reports. $2015 ;(79): 1-16$.

2. Chan M, Wu X, Wu J, Wong S, Chung V. Safety of Acupuncture. Scientific Report. 2017; 7: 1-11

3. Organización Mundial de la Salud. Estrategias de OMS sobre medicina tradicional 2014-2023. Ginebra: OMS; 2013. Disponible en: http://apps.who.int/medicinedocs/documents/s2120 1es/s21201es.pdf [Consultado el 20-01-2018].

4. Muñoz-Ortego J, Solans-Domènech $\mathrm{M}$, Carrion $\mathrm{C}$. Indicaciones médicas de la acupuntura: revisión sistemática. Medicina Clínica. 2016; 147(6):250-256.

5. Litscher G, Rong P. Auricular acupuncture. Evidencebased Complementary and Alternative Medicine: eCAM. 2016; 2016:4231260.

6. Stanton G. Auricular acupuncture as an alternative medicine. HSOA Journal of Alternative, Complementary \& Integrative Medicine. 2017;3:1-3.

7. MINSAL. Encuesta Nacional de Salud 2016-2017. Chile: Ministerio de Salud de Chile; 2017.

8. Heymsfield S, Wadden T. Mechanism, Pathophysiology, Managment of Obesity. The new England Journal of Medicine. 2017;376:254-266.

9. Calderon C, Forns M, Varea V. Implicación de la ansiedad y la depresión en los trastornos de alimentación de jóvenes con obesidad. An Pediatr 2009;71(6):489-94.

10. Da Silva A. El tratamiento de la ansiedad por intermedio de la acupuntura: un estudio de caso. Psicol. Cienc. Prof. 2010; 30(1):200-211.

11. Haddad M, Marcon S. Acupuntura y apetito de trabajadores obesos de un hospital universitario. Acta. Paul. Enferm. 2011; 24(5):676-682.

12. Set T, Cayir Y, Guven A. Effects of ear acupunture therapy for obesity on the depression of obese women. Acupunct. Med. 2014; 32:427-429.

13. Viner RM, Hsia Y, Tomsic T, Wong IC. Efficacy and safety of anti-obesity drugs in children and adolescents: systematic review and meta-analysis. Obes Rev. 2010 Aug; 11(8):593-602.

14. Oleson T. Auriculotherapy Manual: Chinese and Western Systems of Ear Acupuncture, Ed 3. London: Churchill Livingstone; 2003.

15. Schenider H, Esbitt S, González J. Hamilton anxiety rating scale. In: Gellman $M$, Turner J. (edits). Encyclopedia of Behavioral Medicine. New York: Springer; 2013.

16. World Health Organization. Obesity: preventing and managing the global epidemic. Report of a WHO Consultation. WHO Technical Report Series 894. Geneva: WHO; 2000. 
17. Consejo de Organizaciones Internacionales de las Ciencias Médicas/Organización Mundial de la Salud. Pautas éticas internacionales para la investigación biomédica en seres humanos. Ginebra: CIOMS/OMS; 2002. Disponible en: http://www.ub.es/rceue/archivos/Pautas_Eticas_Inter nac.pdf

18. Fischer F, Lewith G, Witt C, Linde K, Von Ammon K, Cardini $F$, et al. High prevalence but limited evidence in complementary and alternative medicine: guidelines for future research. BMC Complementary and Alternative Medicine. 2014;14:46.

19. Clarke TC, Black LI, Stussman BJ, Barnes PM, Nahin RL. Trends in the Use of Complementary Health Approaches Among Adults: United States, 2002-2012. National health statistics reports. 2015;(79):1-1.

20. Li X. Complementary and alternative medicine for treatment of food allergy. Immunol Allergy Clin. N. Am. 2018; 38:103-124.

21. Miyuki K, Sato L, Paes M. Auriculotherapy effectiveness in the reduction of anxiety in nursing students. Rev. Esc. Enferm USP. 2012:46(5):1200-1206.

22. Klausenitz C, Hacker H, Hesse T, Kohlmann T, Endlich K, Hahnenkamp K, Usichenko T. Auricular Acupuncture for Exam Anxiety in Medical Student- A Randomized Crossover investigation. PLoS ONE. 2016;11(12):1-9.

23. Gariepy G, Nitka D, Schimitz N. The association between obesity and anxiety disorders in the population: a systematic review and meta-analysis. International Journal of Obesity. 2010;34:407-419.

24. Chukwuononso E. Association between anxiety and obesity_ a study of a Young-adult Nigerian population. J. Neurosci. Rural Pract. 2013;4(1):s13-s18.

25. Lykouras L, Michopoulos J. Anxiety disorders and obesity. Psychiatriaki. 2011;22(4): 307-13.

26. Abdi H, Tayefi M, Reza S, Zhao B, Rayaz M, Ardabili H, et al. Abdominal and auricular acupuncture reduces blood presure in hypertensive patients. Complementary Therapies in Medicine. 2017;31:20-26.

27. Lacey JM, Tershakovec AM, Foster GD. Acupuncture for the treatment of obesity: a review of the evidence. International. Journal of Obesity. 2003;27:419-427.

28. Silva L, Blondet V, Chaves D, Augusta S. Niveles de iones sérica y su relación con los síntomas del síndrome premenstrual en mujeres jóvenes. Nutrición Hospitalaria. 2013;28(6):2194-2200.

29. Hsu C, Wang CJ, Hwang KC, Lee Ty, Chou P, Chang HH. The effect of auricular acupuncture in obese women: a randomized controlled trial. J. Womens Health (Larchmt). 2009;18(6):813-8.

30. Lien CY, Liao LL, Choud $P$ and Hsu CH. Effects of auricular stimulation on obese women: A randomized, controlled clinical trial. European Journal of Integrative Medicine. 2012;4:E45-E53.

31. Ruan Z, Xiang Y, Li J, Zhow X, Huang Z, Dong C. Auricular acupuncture for obesity: a systematic review and meta-analysis. Int. J. Clin. Exp. Med. 2016;9(2):1772-1779.

32. Ito H, Yamada O, Kira Y, Tanaka T, Matsuoda R. The effects of auricular acupuncture on weight reduction and feeding-related cytokines: a pilot study. BMJ open gastroenterology. 2015;2(1):1-7.

33. Yeo S, Kim KS, Lim S. Randomised clinical trial of five ear acupuncture points for the treatment of overweight people. Acupunct. Med 2014; 32: 132-138.

34. Darbandi M, Darbandi S, Mobarhan MG, Owji AA, Zhao $B$, Iraji $\mathrm{K}$, et al. Effects of auricular acupressure combined with low-calorie diet on the leptin hormone in obese and overweight Iranian individuals. Acupunct Med. 2012;30:208-213.

35. Gücel F, Bahar B, Demirtas C, Mit S, Cevik C. Influence of acupuncture on leptin, ghrelin, insulin and cholecystokinin in obese women: a randomized, shamcontrolled preliminary trial. Acupuncture in Medicine. 2012;30:203-207.

36. Kim D, Ham O, Kang C, Jun E. Effects of auricular acupressure using sinapsis alba seeds on Obesity and Self-Efficacy in female College Students. The Journal of Alternative and Complementary Medicine. 2014;20(4):258-264.

37. Chen J, Chen J, Lee S, Mullin G. Potential role for acupuncture in the treatment of food addiction and obesity. Acupunct. Med. 2017;0:1-4. 